\title{
SISMICIDAD DE TACNA 1997
}

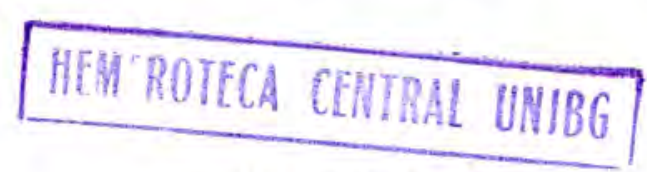

Conrado Jaen Bedoya ${ }^{1}$ Jorge Barriga Gamarra ${ }^{2}$

\author{
R E S U M N N
}

El presente estudio es parte del proyecto "Evaluación Sismica de Tacna", que tiene como objetivo principal conocer el comportamiento sísmico del área de monitoreo de la estación sísmica de la UNJBG, para mitigar los efectos de sismos devastadores. Se detalla la actividad sísmica correspondiente al año 1997, para lo cual se ha elaborado los histogramas de frecuencia-distribución y realizado el análisis e interpretación del record sísmico en forma mensual, con una breve descripción del ambiente sismotectónico y los antecedentes sísmicos de la región, acompañado del correspondiente catálogo sísmico 1997 como resultado del período de observación se han registrado un total de 990 sismos, de los cuales 44 han sido sentidos por la población, estableciéndose que la mayor actividad sísmica se encuentra a más de $100 \mathrm{~km}$. y fundamentalmente entre los 100 y $150 \mathrm{kms}$.

\section{A B S T R A C T}

The current study is part of the "Tacna's Seismicity Evaluation" project, which has as its main objective to know the seismic behavior of the seismic station monitoring area, in order to mitigate the effects of potencially dangerous earthquakes ocurrence. In this study, record of 1997 Tacna seismic activity is detailed. Frecuency and distribution histograms and the monthly analysis and interpretation, with a brief description of the monthly analysis and interpretation have been carried out, with a brief description of the seismotectonic setting and seismic historic record. Seismic catalogue of 1997 data is also provided. As a result of this period of observation 990 earthquakes have been recorded, 44 of them felt with higher intensity than II, and it was clearly defined a higher concentration of the seismic activity located more than $100 \mathrm{~km}$. far from Tacna seismic station, and between 100 and $150 \mathrm{~km}$.

\section{INTRODUCCIÓN}

El proyecto "Evaluación de la Sismicidad de Tacna" fue formulado con la finalidad de conocer la sismicidad de Tacna y sus zonas sismogénicas; para mitigar los efectos de los sismos devastadores como el ocurrido el 12 de agosto de 1868; también para ir ajustando periódicamente los mapas de iso-intensidades; para conocer mejor los suelos sobre los que se encuentran la zona urbana de Tacna y todas aquellas de expansión urbana en razón a la necesidad de contar con vivienda propia, más no por las características de capacidad portante del suelo $u$ otras consideraciones geotécnicas.
El presente trabajo fue realizado en cumplimiento de los siguientes objetivos:

- Emitir el catálogo sísmico y dar pleno cumplimiento a los convenios con el Centro de Investigación Sísmica y Mitigación de Desastres CISMID, Instituto Geofísico del Perú (IGP) y Universidad Nacional de San Agustín (UNSA).

- Conocer la sismicidad de Tacna y sus zonas sismogénicas, para mitigar los efectos de sismos devastadores. 


\section{ANTECEDENTES}

La América del Sur es anualmente escenario de varios eventos sísmicos con magnitudes mayores de 5 ; actividad sísmica que obedece a la dinámica interna de la tierra, originado por el movimiento de las Placas de Nazca hacia la Placa Continental.

El encuentro de estas dos placas, traducido en esfuerzos, deforma a la roca hasta vencer el límite de su elasticidad, fracturando a lo largo de la zona afectada. De esa manera se libera la energia en forma de ondas elásticas de propagación radial respecto al foco o centro sísmico.

La acumulación de energia suficiente para producir la fractura de la roca es lenta, los cálculos estimados por especialistas para este sector de América del Sur, han estimado de 120 a 130 años, el período de retorno de los sismos con magnitudes mayores de 6 .

Considerando la sismicidad histórica de Tacna, se tiene el sismo más devastador ocurrido el 13 de agosto de 1868. La historia sísmica indica que Arica, Tacna, Moquegua e 110 , perdieron casi el $90 \%$ de sus construcciones. De la fecha indicada a la actualidad han transcurrido 130 años y por lo indicado en el párrafo anterior, esta latitud de Sud-América se encuentra a puertas de un sismo con magnitud mayor de 6 a 7 .

Los efectos negativos de un sismo con tal magnitud, serán catastróficos en la actividad socioeconómica de la región y el país.

La Universidad Nacional Jorge Basadre Grohmann, a través del Instituto de Investigación Sismica y Geotecnia, comprometido con las amenazas naturales, viene monitoreando la sismicidad de la región, con la finalidad de definir las zonas sismogénicas y las frecuencias sísmicas de la región. Los resultados permiten conocer el comportamiento sísmico que conllevará a una mejor planificación urbana de los nuevos asentamientos.

\section{UBICACIÓN Y CLIMA}

Tacna es el departamento austral del Perú, ubicado en la frontera Perú-Chile. La ciudad de Tacna se encuentra a una altitud de 450 a 550 msnm, está caracterizado por encontrarse en el desierto de Atacama, el más árido del mundo, con precipitación promedio multianual de 2 a $5 \mathrm{~mm}$ año y temperaturas de 18 a 32 grados en verano y 7 a 27 grados en invierno.
Tacna está intercomunicada con el resto del país y el mundo, mediante la carretera Panamericana que bordea la costa de América del Sur y el aeropuerto Carlos Ciriani.

\section{AMBIENTE SISMOTECTÓNICO}

La región andina es una de las más activas regiones sísmicas del mundo, donde la interacción de la Placa Continental Sudamericana con la Placa Oceánica Nazca es responsable de la mayor parte del tectonismo que produce actividad sismica y volcánica.

El proceso de subducción del margen continental del Perú ha estado actuando desde los comienzos del Jurásico. La mayor expresión morfológica de la zona de subducción es la fosa Perú-Chile, caracterizada en la actualidad por una velocidad convergente de aproximadamente 8 a $10 \mathrm{~cm} / a n ̃ o$ (Minster, Jordán 1978).

La información geofísica de las características de deformación de la corteza oceánica y los sedimentos cercanos y en el interior de la fosa han permitido establecer significativas variaciones de norte a sur en lo referente al campo de esfuerzo regional a lo largo de la fosa, caracterizando zonas de compresión normal a la fosa entre las latitudes $1^{\circ} \mathrm{S}-16^{\circ} \mathrm{S}$ al norte y $27^{\circ} \mathrm{S}-40^{\circ} \mathrm{S}$ al sur, mientras que en la zona sur del Perú y norte de Chile, entre las latitudes $16^{\circ} \mathrm{S}-27^{\circ}$ $\mathrm{S}$, el campo de esfuerzo es tensional, originando fallamiento normal en el borde de la placa oceánica (Wortel, Cloethingh 1985).

En la región sur del Perú la sismicidad está mayormente localizada en la Placa Oceánica en proceso de subducción y una actividad considerable en el bloque Litosférico Continental de aproximadamente $300 \mathrm{Km}$. de espesor, con ocurrencia de volcanes y el plano de sismicidad buzante a una profundidad de aproximadamente $150 \mathrm{Km}$. debajo de la línea de volcanes.

En la región central y norte del Perú hay ausencia de volcanes, la sismicidad no va más allá de los 100 $\mathrm{Km}$. de profundidad en la placa oceánica y la distribución focal es más dispersa en la litósfera continental (fig. 1). De acuerdo a S. SACKS (1977) estos patrones de actividad sísmica y volcánica están relacionados a la presencia o ausencia de Astenósfera inelástica entre la placa de subducción y la litósfera continental. 
Las estructuras cuaternarias del ambiente geológico y neotectónico de la frontera continental PerúChile (pliegues, flexuras y fallas), se encuentran ubicadas en fajas que corresponden aproximadamente a límites de las unidades morfoestructurales de la región, siendo las zonas de mayor peligro sismotectónica: la zona costera, el límite entre la Cordillera Occidental y el Pie de Monte Pacífico y en menor grado los ramales cordilleranos cubiertos con glaciares y fallas activas en su cercanía (CERESIS 1985).

De acuerdo a las características descritas la zona de monitoreo de la estación sísmica de UNJBG-Tacna es considerada de alta actividad sísmica y alto potencial para la ocurrencia de grandes terremotos, donde la actividad sísmica continental es de profundidad media $(70 \mathrm{~km}$. $<\mathrm{h}<300 \mathrm{Km}$.) y la mayor parte de la actividad superficial ( $\mathrm{h}<70 \mathrm{Km}$.) está en la porción oceánica donde han ocurrido los más destructivos y catastróficos terremotos y están directamente relacionados al proceso de subducción en la fosa Perú-Chile.

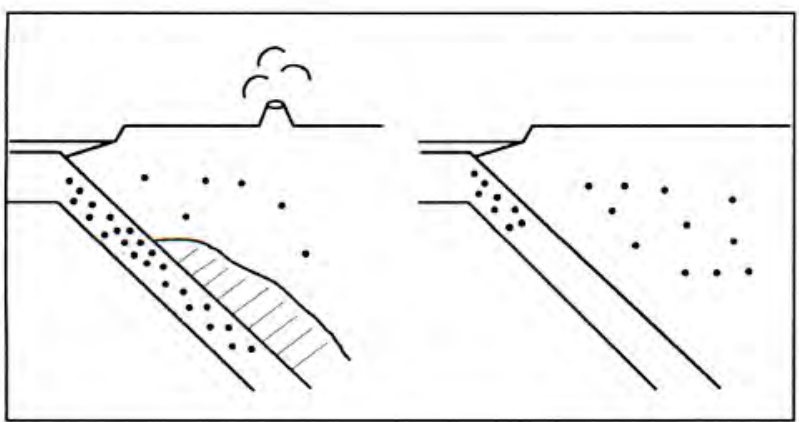

Fig. 1. Esquema de la región Sur del Perú-Chile (izquierda) y la región Central del Perú (derecha). La zona sombreada indica la Astenósfera. Donde ella existe, los sismos (puntos) están principalmente confinados a la placa en subducción y los volcanes ocurren encima a 150 - 175 Km. Donde la Astenósfera estáausente, como en la región Central del Perú, no hay ocurrencia de volcanes y la sismicidad es más dispersa. (S. Sacks 1977).

\section{EL INSTITUTO DE INVESTIGACIÓN SÍSMICA Y GEOTECNIA DE LA UNJBG (ISGE)}

El Instituto de Investigación Sísmica y Geotecnia de la Universidad Nacional Jorge Basadre Grohmann ISGE/UNJBG, viene funcionando desde 1984 y bajo Resolución Rectoral desde 1988.
Durante este período se ha obtenido información importante sobre la sismicidad de Tacna, información que amerita ser procesada e interpretada.

\section{INSTRUMENTAL DEL ISGE}

El Instituto ha iniciado su funcionamiento con la Estación Sísmica conformado por el siguiente instrumental:

Sismómetro: Kinemetries de una sola componente

Sismografo : MEQ 800 con tambor para papel ahumado

Radio : Marca PHYLIPS para control del reloj

Posteriormente el Centro de Investigación Sísmica y Mitigación de Desastres (CISMID), a través de un convenio con el ISGE, instaló un Acelerógrafo Marca SLAVE para registrar todo evento sísmico con magnitud igual o mayor de 3 .

La ubicación geográfica de la Estación Sísmica de la Universidad Nacional Jorge Basadre Grohmann es:

Latitud Sur : $18^{\circ} 09^{\prime} 15.5^{\prime \prime}$
Longitud Oeste : $70^{\circ} 14^{\prime} 51.6^{\prime \prime}$

En 1994, el Instituto Geofísico del Perú, instaló un magnetómetro próximo a la Facultad de Agronomia, con la finalidad de evaluar la variación del magnetismo terrestre, durante el eclipse total de sol, del 03 de noviembre de 1994, instrumento que actualmente se encuentra en ISGE. Dicho equipo ha operado solo tres meses y en la actualidad está paralizado a falta de papel fotográfico.

\section{SISMICIDAD DE TACNA EN 1997}

La actividad sísmica de Tacna, se viene registrando en forma diaria en el ISGE, para éste fin el sismógrafo viene funcionando con una ganancia o sensibilidad de G72, en la escala de 60 a 120 decibiles.

La baja sensibilidad con el que viene trabajando es para evitar enmascaramiento del ruido sísmico a las señales del sismo real en la estación; de esa manera los sismogramas resaltan las señales sísmicas, fundamentalmente aquellas con distancia epicentral no mayor de $200 \mathrm{Km}$. de Tacna. También es importante indicar que la sensibilidad G72 no 
permite registrar la microsismicidad del lugar, que para los fines de la sismicidad tacneña no son de mayor interés.

\section{SISMOGRAMAS}

Son los registros que se obtienen del sismógrafo graduado para 24 horas de registro, con el cambio del papel diario que se realiza entre las 08 y 10 de la mañana.

Para el análisis o lectura de los sismos registrados, previamente se fija con gomalaca el papel ahumado y luego se inicia la lectura de los tiempos de arribo de las ondas, únicamente $\mathrm{P}$ y $\mathrm{S}$, siempre que estas sean legibles.

Posteriormente se calcula la diferencia de tiempos $S$ menos $\mathrm{P}$, diferencia que permite determinar la distancia entre la estación sísmica y el epicentro.

Esta actividad se realiza a diario y en caso de ocurrir un sismo perceptible, pasado el evento, de inmediato se procede a la lectura, previo enlacado del sismograma; esta información es comunicada a, Defensa Civil, para la toma de decisiones y el envio de auxilio si así lo amerita el caso.

\section{ANÁLISIS EINTERPRETACIÓNDELA SISMICIDAD 1997}

La información acumulada se interpreta en forma mensual con sus respectivos cuadros.

\section{Enero}

Durante este mes se han registrado 76 sismos, de ellos 02 sentidos con intensidades menores de II en la escala modificada de Mercally o MM. (figs. 2, 3).

La sismicidad registrada, en su mayoría tienen su epicentro más allá de $150 \mathrm{Km}$. de Tacna (fig. 4).

\section{Febrero}

En este mes se registraron 65 sismos, de ellos 03 sentidos con intensidades menores de $3 \mathrm{MM}$., con efectos secundarios sin importancia (figs. 2, 3).

La sismicidad se ha circunscrito en un radio de 100 a 150 kilómetros de Tacna (fig. 4).

Amerita indicar la presencia de sismos lejanos ocurridos a más de 500 kilómetros de Tacna. Por su lejanía no son peligrosos y su importancia gravita en el record estadistico (Ver registro sísmico Feb.).

\section{Marzo}

Durante el mes de Marzo la sismicidad ha continuado en forma normal, registrándose 82 sismos, de los cuales 2 fueron percibidos por los Tacneños con intensidades menores de II (figs. 2, 3).

La mayor actividad sísmica está localizada en un radio menor de $150 \mathrm{Km}$ y mayor de $100 \mathrm{Km}$. de Tacna. (Ver registro sismico, Marzo).

\section{Abril}

La actividad sísmica registrada del mes de Abril, fue la más alta del año, con 124 sismos; 9 sismos sentidos, 2 de ellos causaron temor y desesperación en la población tacneña (figs. 2, 3), por esta razón transcribimos algunas ocurrencias de lo sucedido.

El día 01 de abril de 1997 a las 13 horas, 34 minutos con 31.8 segundos se inició un sismo de intensidad $V$ MM. a $150 \mathrm{Km}$. de Tacna; el sismo se inició violentamente, toda la población se volcó a las calles con mucho temor.

Cuando todo volvió a su normalidad, se inició otro sismo a las 13 horas, 43 minutos con 12.5 segundos, sentido con VI MM. de intensidad mayor que el primero, esto causó mayor confusión que el primero; la desesperación se apoderó de muchos ciudadanos; en algunos barrios la gente salió a las calles buscando lugares seguros para pasar la noche. Otras personas experimentaron alegría por haber ocurrido el sismo que se esperaba desde hace 130 años (13-08-1868). (Ver registro sísmico Abril).

\section{Mayo}

Es el segundo mes del año con mayor actividad sísmica. Se han registrado 94 sismos, sólo 2 sentidos con intensidad menor de II MM. Como en los 3 últimos meses, la mayor actividad sísmica está centrada entre 100 a 150 Km. de Tacna. (figs. 2, 3, 4).

\section{Junio}

Sexto mes del año, se ha registrado 87 sismos, de ellos sólo 3 fueron sentidos por la población tacneña, con intensidades menores de II MM.

La mayor actividad sísmica se encuentra dentro de los $150 \mathrm{~km}$. a la redonda. (figs. 2, 3, 4 y registro sísmico correspondiente). 


\section{Julio}

La sismicidad de este mes fue menor que el de los meses anteriores, como muestra el cuadro estadístico de sismos.

Se han registrado 70 sismos, 3 de ellos sentidos por la población con intensidad de II (figs. 2, 3).

Otra característica que se nota es el buen número de sismos lejanos registrados con distancia epicentral mayor de $300 \mathrm{Km}$. (fig. 4).

\section{Agosto}

La sismicidad de este mes se incrementó respecto a los meses anteriores, característica normal para este mes, por lo menos para el Sur Peruano (Arequipa y Tacna).

Se han registrado 90 sismos, con solo 2 de ellos percibidos ambos con intensidad de III en la escala MM.

Durante el mes no hubo ningún movimiento sísmico los dias 17,21 y 22. (figs. 2, 3, 4).

\section{Setiembre}

En este mes se han registrado 81 sismos en total, siete de ellos perceptibles, con intensidades menores de 3 MM. (figs, 2, 3).

Como en los anteriores meses, la mayor actividad sísmica se encuentra a más de $100 \mathrm{~km}$. de Tacna. (fig. 4).

\section{Octubre}

La estadística sísmica del mes de octubre ha sufrido algunas variaciones, por fallas y mantenimiento del sismógrafo. Durante los días operados se ha registrado 58 sismos, 5 de ellos, percibieron con intensidades menores de III en la escala MM. Siguiendo el patrón de la sismicidad de Tacna, la mayor actividad se encuentra fuera de los $100 \mathrm{~km}$. de la estación sísmica (Ver registro sísmico Octubre y figs. $2,3,4)$.

\section{Noviembre}

La actividad sísmica de este mes está dentro de lo normal con 83 sismos registrados y tres de ellos percibidos con intensidades menores de $3 \mathrm{MM}$. Respecto a la distancia epicentral de los sismos, sigue el patrón más alla de $100 \mathrm{~km}$. de Tacna, registrándose cinco eventos sísmicos a menor distancia de $100 \mathrm{~km}$. (figs. 2, 3, 4).

\section{Diciembre}

Durante este mes se han registrado 80 sismos, 03 de ellos sentidos por la población con intensidades menores de III en la escala MM. (Ver rgistro sísmico Diciembre).

La mayor actividad sísmica viene registrándose más allá de $100 \mathrm{Km}$.; dentro del radio mencionado se registraron 12 sismos (fig. 4).

\section{CONCLUSIONES}

- La sismicidad registrada en Tacna durante el año de 1997 fue de la siguiente manera:

Sismos registrados durante el año, 990 en total.

Sismos sentidos por la población, 44 .

Sismos con distancia epicentral menor de $100 \mathrm{Km}$., 481.

Sismos con distancia epicentral mayor de $100 \mathrm{Km}$., 381.

- La mayor actividad sísmica en Tacna, se encuentra a más de $100 \mathrm{Km}$., fundamentalmente entre 100 y $150 \mathrm{Km}$.

- La sensibilidad con el que opera el sismográfo de la estación sísmica G72, con finalidad de no registrar el ruido sísmico, no permite registrar la microsismicidad de Tacna, detalle sin mayor repercusión en el control estadístico de los sismos en esta región.

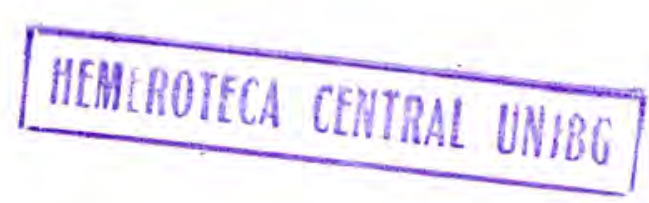


Fig. 2. Actividad Sismica. Enero - Diciembre 1997.

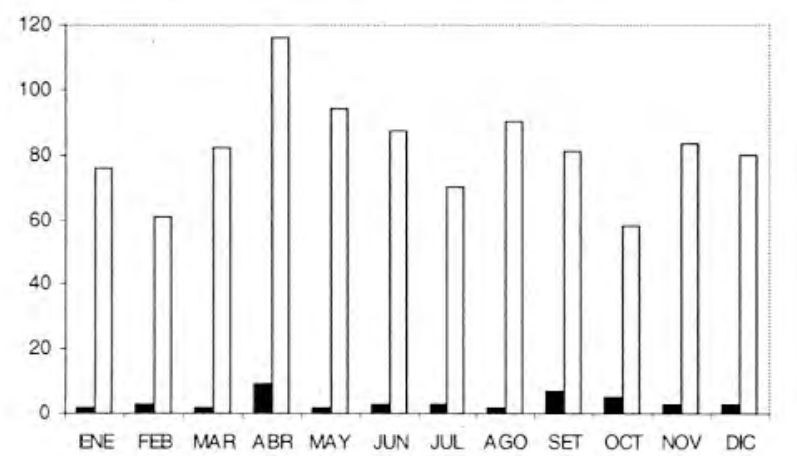

- SISMOS SENTIDOS 口 SISMOS NO SENTIDOS
Fig. 3. Intensidad de sismos percibidos por la población.

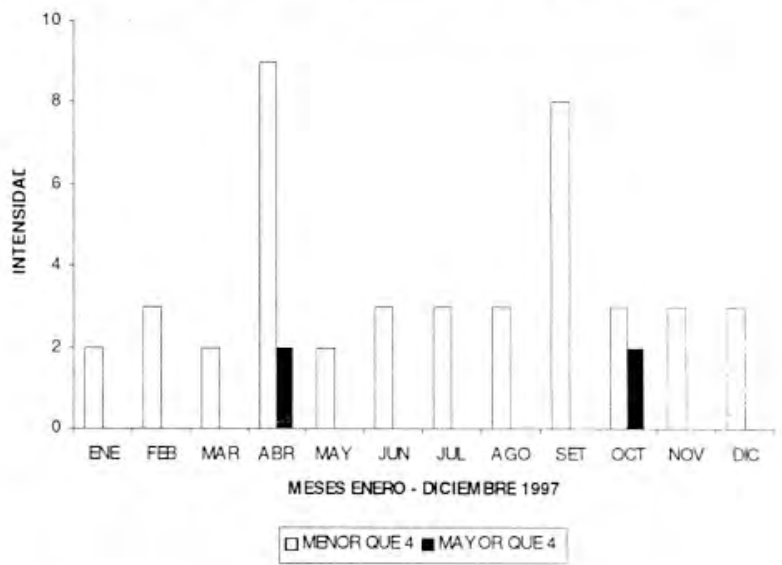

Fig. 4. Distancia Epicentral. Enero - Diciembre 1997.

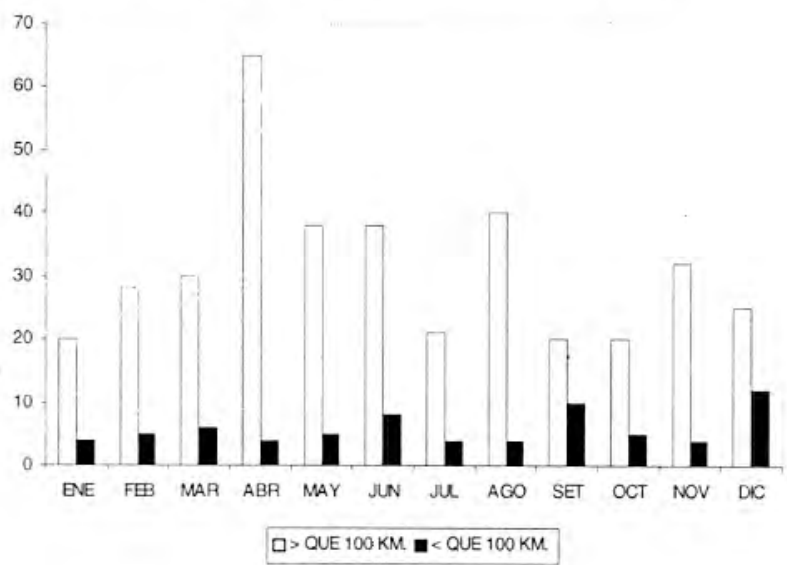

\section{REFERENCIAS BIBLIOGRÁFICAS}

CERESIS (1985) Memoria descriptiva "Mapa Neotectónico Preliminar para América del Sur" Proyecto SISRA, Volumen 11 Lima - Perú.

DAM/GINEBRA, INDECI (1995) "Reducción de los Efectos Destructores de los Sismos en los Departamentos de Arequipa, Moquegua y Tacna" . Folleto preparado por DDH/GINEBRA - INDECI.

E.K. WARSI, W.C. Hilde (1990) "Convergence Tectonics and Sediment Subduction Proces Along The Perú Trench". Memorias del Pacific Rim 90 Cengres Australia.
I SELWYN SACKS (1977). "Interrelationship Between Volcanism, Seismicity, and anelasticity in Western South America", Tectonophysics, 37131 - 139 Ansterdam The Netherlands.

INSTITUTO GEOFISICO DEL PERU. "Catálogo Sísmico SISAN 1984"

J. ALVA HURTADO, L.A. CASAVERDE Y OTROS "Catálogo Sísmico del Perú" 1985.

M.J.R. WORTEL, S.A.PL. CLOETINGH (1985) "Accretion and Lateral Variations in tectonic Structure along The Perú-Chile Trench". Tectonophysics, 112 (1985) 443 462, Amsterdam The Netherlands. 